\title{
SCN5A: the greatest HITS collection
}

\author{
David S. Park and Glenn I. Fishman
}

Leon H. Charney Division of Cardiology, New York University School of Medicine, New York, New York, USA.

\begin{abstract}
Heart failure (HF) has been referred to as the cardiovascular epidemic of our time. Understanding the molecular determinants of HF disease progression and mortality risk is of utmost importance. In this issue of the $J C l$, Zhang et al. uncover an important link between clinical HF mortality risk and a common variant that regulates SCN5A expression through microRNAdependent (miR-dependent)mechanisms. They also demonstrate that haploinsufficiency of SCN5A is associated with increased accumulation of reactive oxygen species (ROS) in a genetically engineered murine model. Their data suggest that even modest depression of SCN5A expression may promote pathologic cardiac remodeling and progression of HF.
\end{abstract}

modulate SCN5A expression by altering miR-dependent regulation. MiRs are short ( 19-22 nucleotides), noncoding RNA species that are potent regulators of mRNA transcript stability and translation (21, 22). MiRs are encoded either in intronic regions where they are cotranscribed with protein-coding exons, or in intergenic regions under the control of their own promoters $(21,22)$. Mature miRs are coassembled with Argonaute (Ago) proteins as part of the RNA-induced silencing complex (RISC) (21). In the classic paradigm, miRs target mRNA via their 3' untranslated region (UTR) through complementary base-pair interaction with the miR "seed" region ( 5 ' region of miR; nucleotide positions 2-8). This miR-mRNA interaction targets mRNA for cleavage or reduces translational efficiency (21). The short sequence interactions allow for miRs to broadly regulate an array of mRNA transcripts that collectively control biological processes, such as calcium homestasis (23) or metabolism (24). Therefore, identification of bona fide miR targets in a given tissue is essential for therapeutic targeting and prevention of off-target effects.

To identify miR targets in the heart, Boudreau and colleagues previously reported a high throughput method to globally profile miR-mRNA target interactions in human cardiac tissue (25). They applied a technique known as high-throughput sequencing of RNA isolated by crosslinking immunoprecipitation (HITS-CLIP), where Ago2 protein was immunoprecipitated to identify bound miRs and mRNAs $(25,26)$. By overlaying common SNPs on top of the transcriptome-wide map of miR binding sites, they identified a non-amino acid altering (synonymous) SNP (rs1805126, $\mathrm{T}>\mathrm{C}$ ) adjacent to a miR-24 interaction site in the SCN5A coding sequence (25). The rs1805126 minor allele was previously shown to associate with cardiac conduction parameters in GWAS (18), but the synonymous nature of the variant led to the presumption that the variant was not causal.

Zhang et al. (20) hypothesized that the rs1805126 minor allele alters miR-24- 
A SCN5A rs1805126 (major allele)

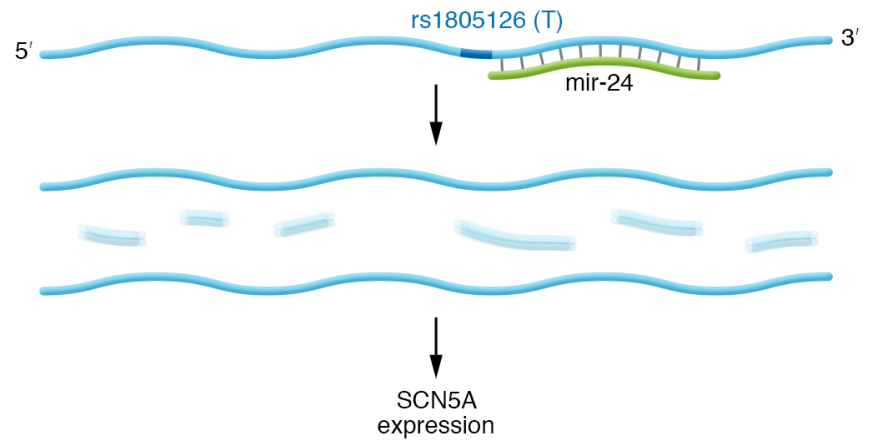

B SCN5A rs1805126 (minor allele)

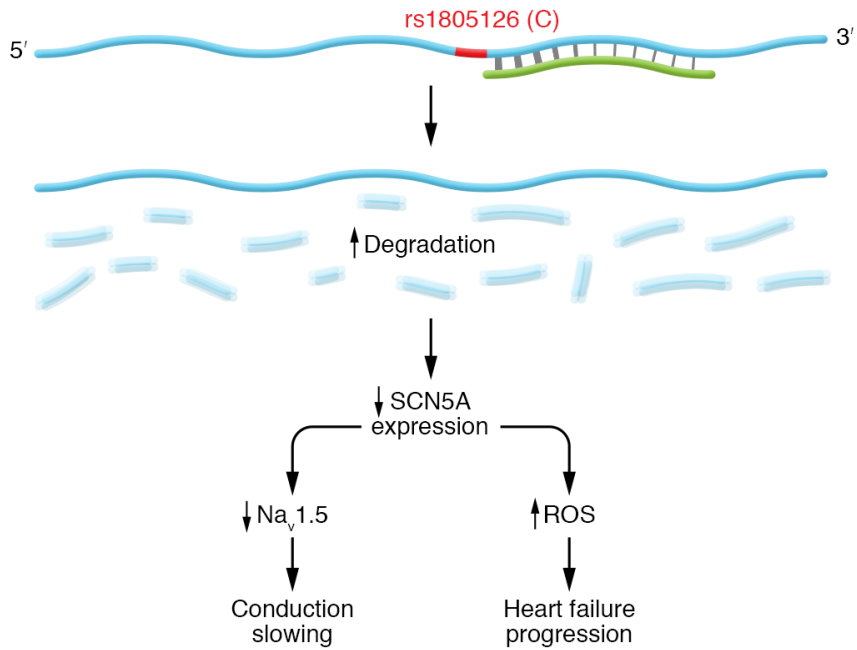

Figure 1. Proposed link between microRNA-dependent regulation of SCN5A and disease progression. Genome-wide association studies (GWAS) have linked a synonymous SNP (rs1805126) in the SCN5A gene with electrocardiographic measures. (A) Ago2 HITS-CLIP data identify a microRNA-24 (miR-24) binding site immediately adjacent to this SNP. (B) Probability of Interaction by Target Accessibility (PITA) analysis indicates that the rs1805126 minor allele (C) is a thermodynamically more favorable miR-24 target compared to the major allele (T), resulting in greater SCN5A degradation and diminished $\mathrm{Na}_{\mathrm{v}} 1.5$ expression. While conduction slowing is a predictable consequence of diminished sodium channel expression, Zhang and colleagues (22) show, surprisingly, that reduced SCN5A expression is also associated with increased myocardial reactive oxygen species (ROS), and suggest that ROS accumulation promotes heart failure progression and increased nonarrhythmic mortality.

dependent regulation of SCN5A (Figure 1). As the rs1805126 variant does not alter the "seed" interaction sequence, the authors speculated and tested through computational means that the $\mathrm{C}$ allele produced a more favorable miR-24 interaction with the SCN5A transcript. In human heterologous expression systems, miR-24 suppressed $\mathrm{Na}_{\mathrm{v}} 1.5$ expression more significantly with the SCN5A C allele versus T allele. MiR24 mimics also reduced sodium current density in neonatal rat cardiomyocytes (NRCMs). In human heart samples, presence of the rs1805126 CC genotype was associated with lower levels of SCN5A mRNA and $\mathrm{Na}_{\mathrm{v}} 1.5$ channel protein compared to the TT genotype, whereas miR-24 expression was similar between groups. Similar results were seen in expression quantitative trait loci (eQTL) studies (27, 28) and analysis of hearts heterozygous for rs1805126, where the $\mathrm{C}$ allele was associated with lower SCN5A mRNA levels.

\section{Clinical consequences of SCN5A modulation}

To explore the clinical consequences of the rs1805126 variant in HF patients, Zhang et al. (20) examined the effect of rs1805126 genotypes in cardiomyopathy patients with ICDs from the Genetic Risk Assessment of Defibrillator Events (GRADE) study
(29) and found a higher mortality rate in patients homozygous for the $\mathrm{C}$ allele. Surprisingly, the rs1805126 genotype did not significantly associate with appropriate ICD therapies (a surrogate marker of arrhythmic death). In addition, the authors did not find a significant association with electrocardiographic parameters, although the study was underpowered to do so. As previously stated, prolonged QRS duration is associated with increased morbidity and mortality in HF patients due to dyssynchronous myocardial contraction; therefore, it will be important to reanalyze this association with increased patient enrollment or in another dataset, especially given the known association of rs1805126 with cardiac conduction parameters (18). Similarly, these data should be analyzed for associations between the homozygous rs1805126 genotype and cardiac resynchronization therapy, indicated for patients with significant QRS prolongation and HF (30). Lastly, it would be of interest to note whether the CC genotype is associated with increased right ventricular pacing percentage, as this type of pacing produces myocardial dyssynchrony and has been shown to reduce left ventricular ejection fraction and increase morbidity and mortality (31).

To explore the potential mechanism whereby reduced $S C N 5 A$ expression is associated with a more severe cardiomyopathic phenotype, Zhang et al. (20) studied $S c n 5 a$ heterozygous knockout mice, which develop increased fibrosis at advanced age (32). Scn5a heterozygous knockout hearts had significantly increased reactive oxygen species (ROS) as evidenced by an approximately 2.5 -fold increase in oxidation of dihydroethidium (DHE, a measure of steady-state levels of superoxide), which was evident before fibrotic changes. Although association between rs1805126 genotype and increased oxidative stress was not examined, the finding that reduced $S c n 5 a$ expression is sufficient to increase ROS is intriguing and needs further investigation. Accumulation of ROS can result from either overproduction or impaired clearance (33). Increased ROS production in $\mathrm{HF}$ is primarily due to functional uncoupling of the mitochondrial electron transport chain (34-37); however, other sources include xanthine oxidase (38), nitric oxide synthase (39), cyclooxygenase (40), and NAD(P)H oxidases (41). Mechanisms of impaired clearance by antioxidants include reduced activity of superoxide dismutase (42) and catalase (43). Identifying which of these pathways contributes to ROS accumulation due to reduced $\mathrm{Na}_{\mathrm{v}} 1.5$ expression will be an important first step 
in deciphering the mechanisms underlying induction of profibrotic pathways and the development of cardiomyopathy.

\section{Concluding remarks}

The work of Zhang et al. (20) adds to the growing body of evidence that sequence variants that regulate $S C N 5 A$ expression can have significant consequences on HF disease progression and mortality. Although the mechanism of worsening HF associated with rs1805126 will need further evaluation, these findings bring us one step closer to creating a genetic HF risk score, which can be used to personalize therapies for this complex and growing patient population.

\section{Acknowledgments}

This work is supported by grants from the NIH to DSP (R01 HL132073), and GIF (R01 HL105983 and R01 HL92727), an American Heart Association Scientist Development Grant (17SDG33411201) to DSP, and a Fondation LeDucq Transatlantic Network Award to GIF.

Address correspondence to: Glenn I. Fishman, Leon H. Charney Division of Cardiology, NYU School of Medicine, 522 First Avenue, Smilow 801, New York, New York 10016, USA. Phone: 212.263.3967; Email: glenn.fishman@nyumc.org.

1. Metra M, Teerlink JR. Heart failure. Lancet. 2017;390(10106):1981-1995

2. Nattel S, Maguy A, Le Bouter S, Yeh YH. Arrhythmogenic ion-channel remodeling in the heart: heart failure, myocardial infarction, and atrial fibrillation. Physiol Rev. 2007;87(2):425-456.

3. Iuliano S, Fisher SG, Karasik PE, Fletcher RD, Singh SN, Department of Veterans Affairs Survival Trial of Antiarrhythmic Therapy in Congestive Heart Failure. QRS duration and mortality in patients with congestive heart failure. $\mathrm{Am}$ Heart J. 2002;143(6):1085-1091.

4. Kashani A, Barold SS. Significance of QRS complex duration in patients with heart failure. $\mathrm{JAm}$ Coll Cardiol. 2005;46(12):2183-2192.

5. Park DS, et al. Genetically engineered SCN5A mutant pig hearts exhibit conduction defects and arrhythmias. J Clin Invest. 2015;125(1):403-412.

6. Papadatos GA, et al. Slowed conduction and ventricular tachycardia after targeted disruption of the cardiac sodium channel gene Scn5a. Proc Natl Acad Sci U S A. 2002;99(9):6210-6215.

7. Park DS, et al. Fhf2 gene deletion causes temperature-sensitive cardiac conduction failure. Nat Commun. 2016;7:12966.

8. Shekhar A, et al. Transcription factor ETV1 is essential for rapid conduction in the heart. JClin
Invest. 2016;126(12):4444-4459.

9. Schott JJ, et al. Cardiac conduction defects associate with mutations in SCN5A. Nat Genet. 1999;23(1):20-21.

10. Chen Q, et al. Genetic basis and molecular mechanism for idiopathic ventricular fibrillation. Nature. 1998;392(6673):293-296.

11. Bennett PB, Yazawa K, Makita N, George AL Jr. Molecular mechanism for an inherited cardiac arrhythmia. Nature. 1995;376(6542):683-685.

12. Wang Q, et al. SCN5A mutations associated with an inherited cardiac arrhythmia, long QT syndrome. Cell. 1995;80(5):805-811.

13. Olson TM, et al. Sodium channel mutations and susceptibility to heart failure and atrial fibrillation. JAMA. 2005;293(4):447-454.

14. Wan E, et al. Aberrant sodium influx causes cardiomyopathy and atrial fibrillation in mice. J Clin Invest. 2016;126(1):112-122.

15. Shang LL, et al. Human heart failure is associated with abnormal C-terminal splicing variants in the cardiac sodium channel. Circ Res. 2007;101(11):1146-1154.

16. Sotoodehnia $\mathrm{N}$, et al. Common variants in 22 loci are associated with QRS duration and cardiac ventricular conduction. Nat Genet 2010;42(12):1068-1076.

17. Pfeufer A, et al. Genome-wide association study of PR interval. Nat Genet. 2010;42(2):153-159.

18. Holm H, et al. Several common variants modulate heart rate, PR interval and QRS duration. Nat Genet. 2010;42(2):117-122.

19. Chambers JC, et al. Genetic variation in SCN1OA influences cardiac conduction. Nat Genet. 2010;42(2):149-152.

20. Zhang X, et al. A common variant alters $S C N 5 A-$ miR-24 interaction and associates with heart failure mortality. JClin Invest. 2018;128(3):1154-1163.

21. Ha M, Kim VN. Regulation of microRNA biogenesis. Nat Rev Mol Cell Biol. 2014;15(8):509-524.

22. Quiat D, Olson EN. MicroRNAs in cardiovascular disease: from pathogenesis to prevention and treatment. J Clin Invest. 2013;123(1):11-18.

23. Harada M, Luo X, Murohara T, Yang B, Dobrev D, Nattel S. MicroRNA regulation and cardiac calcium signaling: role in cardiac disease and therapeutic potential. Circ Res. 2014;114(4):689-705

24. Hathaway QA, Pinti MV, Durr AJ, Waris S, Shepherd DL, Hollander JM. Regulating MicroRNA expression: at the heart of diabetes mellitus and the mitochondrion [published online ahead of print October 6, 2017]. Am J Physiol Heart Circ Physiol. https://doi. org/10.1152/ajpheart.00520.2017.

25. Spengler RM, et al. Elucidation of transcriptomewide microRNA binding sites in human cardiac tissues by Ago2 HITS-CLIP. Nucleic Acids Res. 2016;44(15):7120-7131

26. Chi SW, Zang JB, Mele A, Darnell RB. Argonaute HITS-CLIP decodes microRNA-mRNA interaction maps. Nature. 2009;460(7254):479-486.

27. Liu Y, et al. RNA-Seq identifies novel myocardial gene expression signatures of heart failure. Genomics. 2015;105(2):83-89.

28. Koopmann TT, et al. Genome-wide identification of expression quantitative trait loci (eQTLs) in human heart. PLoS One. 2014;9(5):e97380.

29. AlJaroudi WA, et al. Effect of angiotensin- converting enzyme inhibitors and receptor blockers on appropriate implantable cardiac defibrillator shock in patients with severe systolic heart failure (from the GRADE Multicenter Study). Am J Cardiol. 2015;115(7):924-931.

30. Russo AM, et al. ACCF/HRS/AHA/ASE/HFSA/ SCAI/SCCT/SCMR 2013 appropriate use criteria for implantable cardioverter-defibrillators and cardiac resynchronization therapy: a report of the American College of Cardiology Foundation appropriate use criteria task force, Heart Rhythm Society, American Heart Association, American Society of Echocardiography, Heart Failure Society of America, Society for Cardiovascular Angiography and Interventions, Society of Cardiovascular Computed Tomography, and Society for Cardiovascular Magnetic Resonance. J Am Coll Cardiol. 2013;61(12):1318-1368.

31. Sharma AD, et al. Percent right ventricular pacing predicts outcomes in the DAVID trial. Heart Rhythm. 2005;2(8):830-834.

32. Royer A, et al. Mouse model of SCN5A-linked hereditary Lenègre's disease: age-related conduction slowing and myocardial fibrosis. Circulation. 2005;111(14):1738-1746.

33. Moris $\mathrm{D}$, et al. The role of reactive oxygen species in myocardial redox signaling and regulation. Ann Transl Med. 2017;5(16):324.

34. Zorov DB, Juhaszova M, Sollott SJ. Mitochondrial ROS-induced ROS release: an update and review. Biochim Biophys Acta. 2006;1757(5-6):509-517.

35. Sawyer DB, Colucci WS. Mitochondrial oxidative stress in heart failure: "oxygen wastage" revisited. Circ Res. 2000;86(2):119-120.

36. Liu M, Liu H, Dudley SC Jr. Reactive oxygen species originating from mitochondria regulate the cardiac sodium channel. Circ Res. 2010;107(8):967-974.

37. Ide $\mathrm{T}$, et al. Mitochondrial DNA damage and dysfunction associated with oxidative stress in failing hearts after myocardial infarction. Circ Res. 2001;88(5):529-535.

38. Berry CE, Hare JM. Xanthine oxidoreductase and cardiovascular disease: molecular mechanisms and pathophysiological implications. JPhysiol. 2004;555(pt 3):589-606.

39. Umar S, van der Laarse A. Nitric oxide and nitric oxide synthase isoforms in the normal, hypertrophic, and failing heart. Mol Cell Biochem. 2010;333(1-2):191-201.

40. Kim JW, et al. Gene expression of cyclooxygenase in the aging heart. J Gerontol A Biol Sci Med Sci. 2001;56(8):B350-B355.

41. Kuroda J, Ago T, Matsushima S, Zhai P, Schneider MD, Sadoshima J. NADPH oxidase 4 (Nox4) is a major source of oxidative stress in the failing heart. Proc Natl Acad Sci U S A 2010;107(35):15565-15570.

42. Fukai T, Folz RJ, Landmesser U, Harrison DG. Extracellular superoxide dismutase and cardiovascular disease. Cardiovasc Res. 2002;55(2):239-249.

43. Borchi E, et al. Enhanced ROS production by NADPH oxidase is correlated to changes in antioxidant enzyme activity in human heart failure. Biochim Biophys Acta. 2010;1802(3):331-338. 